\title{
Detection of serum anti-melanocyte antibodies and identification of related antigens in patients with vitiligo
}

\author{
M.C. Zhu, C.G. Liu, D.X. Wang and Z. Zhan \\ Clinical Laboratory Center, Air Force General Hospital of PLA, Beijing, China \\ Corresponding author: M.C. Zhu \\ E-mail: zhumeicai_mc@163.com
}

Genet. Mol. Res. 14 (4): 16060-16073 (2015)

Received August 16, 2015

Accepted October 22, 2015

Published December 7, 2015

DOI http://dx.doi.org/10.4238/2015.December.7.19

\begin{abstract}
We detected autoantibodies against melanocytes in serum samples obtained from 50 patients, including 4 with HBV, with vitiligo and identified the associated membrane antigens. Heat shock protein 70 (HSP70) and anti-tyrosinase-related protein 1 (TRP-1) antibody levels were analyzed. The associated antigens in normal human melanocyte were identified by immunofluorescence. Autoantibodies against melanocyte membrane and cytoplasmic proteins were detected by western blot. Membrane antigens with higher frequencies were identified by protein mass spectrometry. The HSP70 and anti-TRP-1 antibody levels $(\mathrm{N}=70 ; 10$ with HBV) were detected by ELISA. The specific antigens were detected in melanocyte cytoplasm and membrane (40/50; $80 \%$ incidence; western blot). The autoantibodies reacted with several membrane antigens with approximate molecular weights (Mr) of 86,000, 75,000, 60,000, 52,000, and 44,000 (strip positive rates: $36,58,22,2$, and $2 \%$, respectively). Thirty percent of the patients showed the presence of cytoplasmic antigens (Mr: $110,000,90,000,75,000,50,000$, and 400,000; strip positive rates: 12 , $4,12,10$, and $2 \%$, respectively). Fifteen and $5 \%$ of the healthy subjects
\end{abstract}


showed positive expression of membrane and cytoplasmic antigens, respectively. Protein mass spectrometry predicted membrane proteins with $\mathrm{Mr}$ of 86,000 and 75,000 and 60,000 to be Lamin A /C and Vimentin X1, respective. High titers of anti-TRP-1 antibody were detected and showed positive correlation with HSP70 $(r=0.927, P<0.01)$. This study identified a novel membrane antigen associated with vitiligo, which might assist future investigations into autoimmune pathogenesis of vitiligo and formation of autoantibodies. HBV infection was correlated to vitiligo.

Key words: Vitiligo; Melanocytes; Autoantigen; Autoantibody; Hepatitis B virus; Heat shock protein 70

\section{INTRODUCTION}

Vitiligo is a common skin disease with an incidence of $1-2 \%$, which is characterized by the loss of melanocytes (Lu et al., 2004). Although its pathogenesis remains to be clarified, a majority of the experimental evidence favors the autoimmune theory; that is, the existence and killing activity of anti-melanocyte autoantibodies have been confirmed by live cell enzyme-linked immunosorbent assay (ELISA), immunoblotting, and complement-mediated cytolytic experiments (Cui et al.,1993; Li et al., 2000, 2001, 2003; Kemp et al., 2007). Vitiligo-related melanocyte-specific antigens include both cytoplasmic and membrane antigens. The former includes tyrosinase, tyrosinaserelated protein 1 (TRP-1), and TRP-2, while the latter have not been extensively researched or elucidated. In this study, anti-melanocyte autoantibodies were detected through the sequential extraction of melanocyte proteins by immunoblotting; membrane antigens with a high recognition rate were identified by protein spectroscopy, and their properties were further clarified in order to facilitate further research into their function and role in vitiligo pathogenesis. Patients with vitiligo demonstrate certain complications, including autoimmune disease and chronic hepatitis B (HBV) (Zhang et al., 2012). In this study, we evaluated the serum anti-TRP-1 antibody and heat shock protein 70 (HSP70) levels in vitiligo patients with hepatitis B, in order to shed some light on the correlation between $\mathrm{HBV}$ infection and vitiligo pathogenesis.

\section{MATERIAL AND METHODS}

\section{Source of clinical specimens}

A total of 70 blood serum samples (10 with HBV infection) were collected from vitiligo patients hospitalized in the Air Force General Hospital from February to April, 2014. Of the 70 patients, 38 were males and 32 were females, with an average age of 28 years. Twenty-six normal patients (14 males and 12 females) were included as normal controls; there was no significant difference in the age distribution between the control and vitiligo groups. Fasting blood samples were collected from all participants using vacuum tubes; these were centrifuged at $3000 \mathrm{rpm}$ for $10 \mathrm{~min}$ at room temperature. The blood serum was transferred from each blood sample to an Eppendorf tube, and preserved in a $-20^{\circ} \mathrm{C}$ refrigerator. Melanocyte samples from the prepuce tissues of healthy adolescents who underwent circumcision, none with HIV, HBV, or mycoplasma infection, were obtained from the Endoscopic Center of our hospital. 


\section{Instruments and reagents}

M2 melanocyte medium, human melanocyte growth stimulating factors, and Accutase Solution were purchased from Promocell (PromoCell GmbH, Jiangsu Punuo Biotechnology Co., Ltd, Wuxi City, China), while dispase II enzyme and Coomassie brilliant blue were obtained from Sigma-Aldrich (St. Louis, MO, USA), 0.25\% trypsin-EDTA was purchased from Invitrogen, fluorescein isothiocyanate (FITC)-labeled goat anti-human IgG was obtained from Euroimmun, and the fluorescence microscope was purchased from Olympus Corporation (Tokyo, Japan). The membrane protein, nucleoprotein, and cytoplasmic protein extraction kits were purchased from KeyGEN Biotech, while the bicinchoninic (BCA) protein quantification kit and the horseradish peroxidase (HRP)-DAB chromogenic substrate kit were obtained from Tiangen Biotech. The HRP-labeled rabbit anti-human IgG antibody was a product of Bioss, and the human monoclonal antibody ELISA Kit and Human HSP70 ELISA Kit were provided by KainuoBio Corporation. The human hepatitis B surface antigen (HBsAg) ELISA kit was purchased from Wantai (Beijing, China), while the enzyme-labeling measuring instrument was a product of Bio-Rad (Hercules, CA, USA).

\section{Culture of melanocytes from healthy adolescents}

Melanocytes from healthy adolescents using a method described extensively in previous studies (Mulekar, 2003; Wang and Zhu, 2011; Zhou et al., 2013). Briefly, the collected prepuce tissues were sterilized and cut into $5 \times 5-\mathrm{mm}$ slices after the removal of subcutaneous tissue. The tissues were then digested with $0.1 \%$ Dispase II enzyme $(1 \mathrm{mg} / \mathrm{mL})$ at $4^{\circ} \mathrm{C}$ for $14 \mathrm{~h}$. The true epidermis layer was separated and digested in $0.25 \%$ trypsin-EDTA solution for 13 min using a gentle pipette. The digestion was terminated with $10 \%$ fetal bovine serum, and the cell density was adjusted to $5 \times 10^{5} / \mathrm{mL}$. The cells were seeded into M2 medium, and incubated in a $5 \% \mathrm{CO}_{2}$ incubator at $37^{\circ} \mathrm{C}$. The medium was changed every 2 days, and cells were passaged with accutase enzyme.

\section{Indirect immunofluorescence staining}

The cells were stained for immunofluorescence using a method detailed by Zhu et al. (2010). Briefly, 30 serum samples were randomly selected from the vitiligo group, and 10 were selected from the control group. Phosphate-buffered saline (PBS) was set as the blank control. Melanocytes in passage three were seeded to culture dishes at a density of $5 \times 10^{5} / \mathrm{mL}$, and cultured for $24 \mathrm{~h}$. The cells were then fixed with cold methanol, rinsed with PBS, and blocked with $5 \%$ goat serum. The blood serum samples were diluted 10 times and incubated with melanocyte cultures at $37^{\circ} \mathrm{C}$ for $1 \mathrm{~h}$. The cells were again rinsed five times with PBS; FITC-labeled goat antihuman IgG was added to these cells, and incubated at $37^{\circ} \mathrm{C}$ for $30 \mathrm{~min}$. Subsequently, the stained cells were washed and sealed, and observed under a fluorescence microscope.

\section{Extraction of melanocyte soluble antigens}

Soluble antigens expressed by melanocytes at passage four were extracted using a membrane protein, nucleoprotein, and cytoplasmic protein extraction kit, according to the method detailed herein. The cells were rinsed with cold PBS and collected after digestion. One milliliter cold 
reagent A [containing $1 \mathrm{~mL}$ Buffer A, $1 \mu \mathrm{L}$ DTT, $10 \mu \mathrm{L}$ phenylmethylsulfonyl fluoride (PMSF), $1 \mu \mathrm{L}$ protease inhibitors, and $5 \mu \mathrm{L}$ phosphatase inhibitor] was added to the collected cells, and the cells were disrupted by ultrasound treatment. The cells were then vortexed for $10 \mathrm{~s}$ and placed on ice for $20 \mathrm{~min}$; the obtained cell lysate was centrifuged, and the supernatant (containing cytoplasmic proteins) was collected and preserved in a $-80^{\circ} \mathrm{C}$ refrigerator. Reagent $\mathrm{B}$ was then added to the precipitate obtained, in order to extract the nuclear protein. Finally, $0.5 \mathrm{~mL}$ cold reagent $\mathrm{C}(1 \mathrm{~mL}$ Buffer $\mathrm{C}$ contains $1 \mu \mathrm{L}$ DTT, $10 \mu \mathrm{L} \mathrm{PMSF}, 1 \mu \mathrm{L}$ protease inhibitor, and $5 \mu \mathrm{L}$ phosphatase inhibitor) was added to the precipitate; this sample was then vortexed and sonicated for $10 \mathrm{~s}$ each and incubated on ice for $10 \mathrm{~min}$ (with shaking). The samples were subsequently centrifuged for $10 \mathrm{~min}$ in order to collect the membrane protein from the supernatant; this was then preserved at $-80^{\circ} \mathrm{C}$.

\section{Protein quantification}

The samples were diluted five times as per the standard instructions provided with the BCA protein quantification kit; the A value of the samples was detected at $562 \mathrm{~nm}$. The concentration of proteins in each sample was calculated using the standard curve.

\section{Sodium dodecyl sulfate polyacrylamide gel electrophoresis (SDS-PAGE)}

The stacking and separating gel were composed of 4 and $10 \%$ polyacrylamide, respectively. The membrane protein and cytoplasmic protein extracted from melanoma cells were heated with loading buffer $(4 X)$ at $100^{\circ} \mathrm{C}$ for $10 \mathrm{~min}$. The samples were then loaded onto an electrophoresis chamber at room temperature (stable voltage of $80 \mathrm{~V}$ for the stacking gel and 100 $\checkmark$ for the separating gel). The electrophoresed samples were stained with Coomassie brilliant blue R250 and the protein bands were observed under the microscope (OLYMPUS CX22).

\section{Western blot}

Protein bands were transferred from the PAGE to a nitrocellulose membrane in an ice-bath under a steady flow of current $(260 \mathrm{~mA})$ for $2 \mathrm{~h}$. The membrane was blocked with $5 \%$ skimmed milk powder containing TBS ( $0.1 \%$ Tween 20$)$ for $2 \mathrm{~h}$ at room temperature with shaking. The membrane was incubated with the primary antibody (serum samples of vitiligo patients or healthy controls; 1:200 diluted with TBS containing $5 \%$ skimmed milk powder) overnight on a $4^{\circ} \mathrm{C}$ shaking table. The membrane was washed thrice with TBS buffer (10 min each), and subsequently incubated with 1:1000 secondary antibody (HRP-labeled rabbit anti human lgG) for $2 \mathrm{~h}$ on a shaking table. The membrane was washed thrice with TBS buffer (10 min each), and subsequently reacted with HRP-DAB (for a color reaction) in the dark.

\section{Protein identification via mass spectrometry}

The proteins were identified by two-stage mass spectrometry, using a $Q$ Exactive LC/MS system (Thermo Scientific Corporation). The protein bands with a relative molecular mass of $86 \mathrm{x}$ $10^{3}, 75 \times 10^{3}$, and $60 \times 10^{3}$ were cut from the polyacrylamide gel for decolorization, reduction, and trypsin digestion. The samples were then fully dissolved and loaded onto a high-performance liquid chromatography-mass spectrometry (HPLC-MS) chamber. HPLC was conducted using a Diane 
NCS3500 system, while MS was performed on a Q Exactive system. The generated data and the downloaded proteome database (human-refseq-20140303-71465s.fasta; downloaded from NCBI) was analyzed using the Proteome Discoverer software.

\section{Detection of anti-TRP-1 antibody and HSP70}

TRP-1 and HSP70 were detected using an anti-TRP-1 antibody ELISA kit and a human HSP70 ELISA kit. Fifty microliters of different concentrations of the standard protein was added to each standard well. Ten microliters of the sample protein (diluted with $40 \mu \mathrm{L}$ sample dilution buffer) was then added to each sample well. HRP-labeled antigen (or antibody; $100 \mu \mathrm{L}$ ) was added to each well, and the plates were incubated in a $37^{\circ} \mathrm{C}$ incubator for $60 \mathrm{~min}$. The plates were then washed five times before adding $50 \mu \mathrm{L}$ of the substrate into each well. The plate was then incubated for 15 min in the dark at $37^{\circ} \mathrm{C}$. Stop solution $(50 \mu \mathrm{L})$ was then added to terminate the reaction, and the absorbance value of each well was measured at $450 \mathrm{~nm}$.

\section{Statistical analysis}

All data were processed using the SPSS (v.19.0) software platform. The count data are reported as means \pm standard deviation (SD). The levels of expression of the TRP-1 antibody and HSP70 among the different groups was compared by one-way analysis of variance (ANOVA); Dunnett T3 method was used to compare two groups. The Spearman test was used for correlation analysis when the total distribution did not correlate with normal distribution. $\alpha=0.05$ was considered to be statistically significant.

\section{RESULTS}

\section{Detection of vitiligo serum anti-melanocytes by indirect immunofluorescence staining}

Twenty-one of the 30 serum samples (obtained from vitiligo patients) showed strong fluorescence against melanocytes in the cytoplasm (Figure 1). On the other hand, the 10 serum samples from healthy controls displayed no such fluorescence signal (Figure 2).

\section{SDS-PAGE separation of healthy adolescent melanocyte cytoplasmic and membrane proteins}

Cytoplasmic and membrane proteins obtained from melanocytes were loaded onto electrophoretic gels and stained with Coomassie Brilliant Blue R250. The observed protein bands are shown in Figures 3 and 4. More than ten bands of cytoplasmic protein (all with a relative molecular weight $<110 \times 10^{3}$ ), and a lower number of membrane protein bands [relative molecular weight (Mr) distributed at $86 \times 10^{3}, 75 \times 10^{3}$, and $60 \times 10^{3}$ ] were observed.

\section{Detection of the vitiligo serum anti-melanocyte antibody activity by western blot}

The cytoplasmic and membrane proteins extracted from melanocytes (diluted five times) 
showed absorbance values (at $562 \mathrm{~nm}$ ) of 0.468 and 0.438 , and were present in concentrations of 2.29 and $2.07 \mathrm{mg} / \mathrm{mL}$, respectively. Twenty micrograms of the transmembrane protein was analyzed. Among the 50 randomly selected serum samples obtained from vitiligo patients, 40 (80\%) positively expressed the anti-membrane protein antibody (Figure 5); the relative molecular weights of the corresponding antigens were $86 \times 10^{3}, 75 \times 10^{3}, 60 \times 10^{3}, 52 \times 10^{3}$, and $44 \times 10^{3}$ and the positive rate for each band was $36 \%(18 / 50), 58 \%(29 / 50), 22 \%(11 / 50), 2 \%(1 / 50)$, and $2 \%$ $(1 / 50)$. Only three of the 20 serum samples obtained from healthy controls positively expressed the anti-membrane protein antibody; these only interacted with antigens with an $\mathrm{Mr}$ of $86 \times 10^{3}, 75 \times$ $10^{3}$, and $60 \times 10^{3}$. The rate of positive expression of each band was $5 \%(1 / 20), 15 \%(3 / 20)$, and $5 \%$ $(1 / 20)$. On the other hand, 15 of the 50 randomly selected vitiligo serum samples $(30 \%)$ positively expressed the anti-cytoplasmic protein antibody (Figure 6); the $\mathrm{Mr}$ of the corresponding antigens were $110 \times 10^{3}, 90 \times 10^{3}, 75 \times 10^{3}, 50 \times 10^{3}$, and $40 \times 10^{3}$, and the positive rate for each band was $12 \%(6 / 50), 4 \%(2 / 50), 12 \%(6 / 50), 10 \%(5 / 50)$, and $2 \%(1 / 50)$. Only one of the 20 serum samples from healthy controls (5\%) positively expressed the anti-cytoplasmic protein antibody, combining an antigen with an $\mathrm{Mr}$ of $90 \times 10^{3}$.

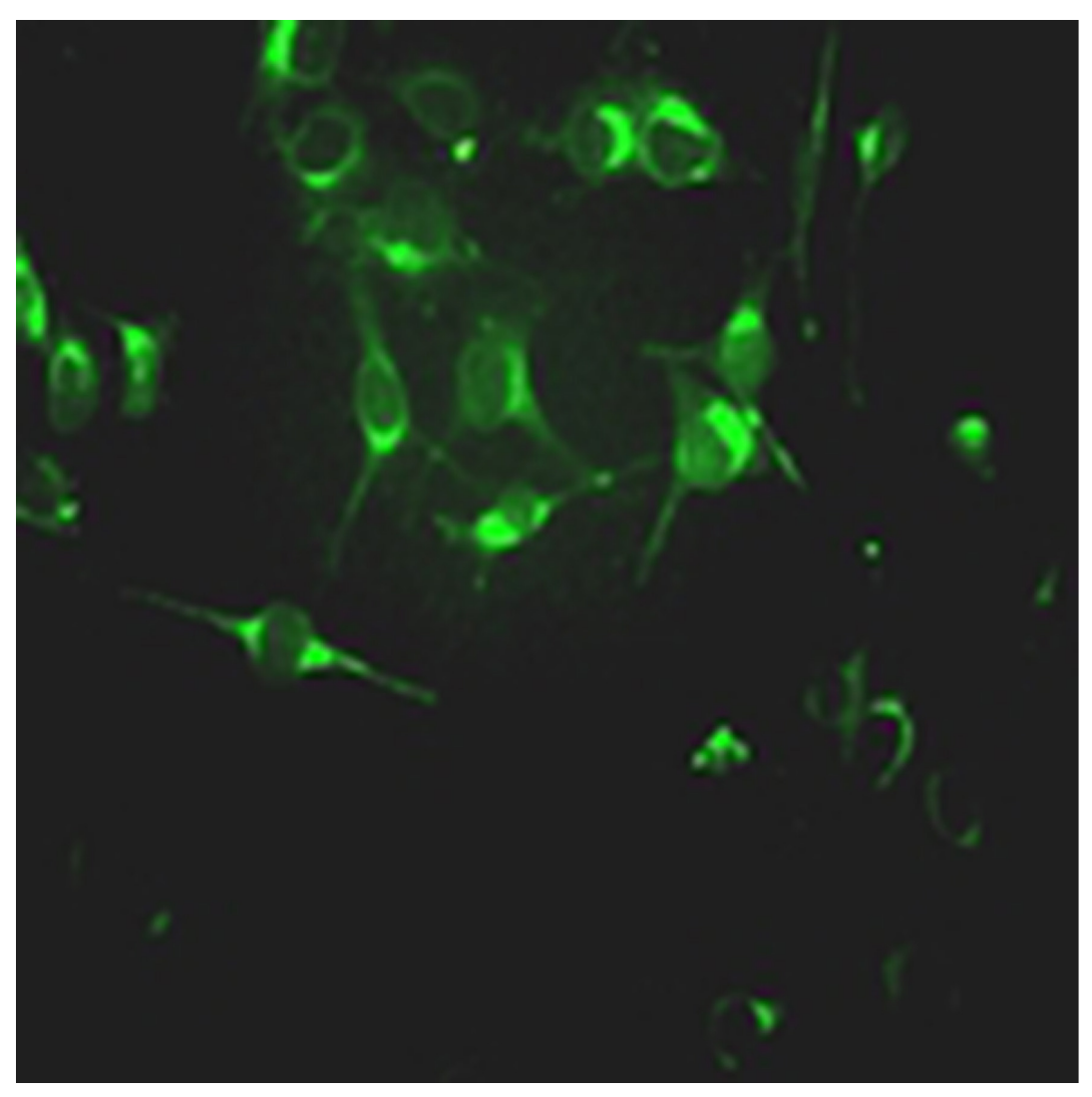

Figure 1. Indirect immunofluorescence staining of melanocytes in serum samples obtained from patients with vitiligo (40X). 


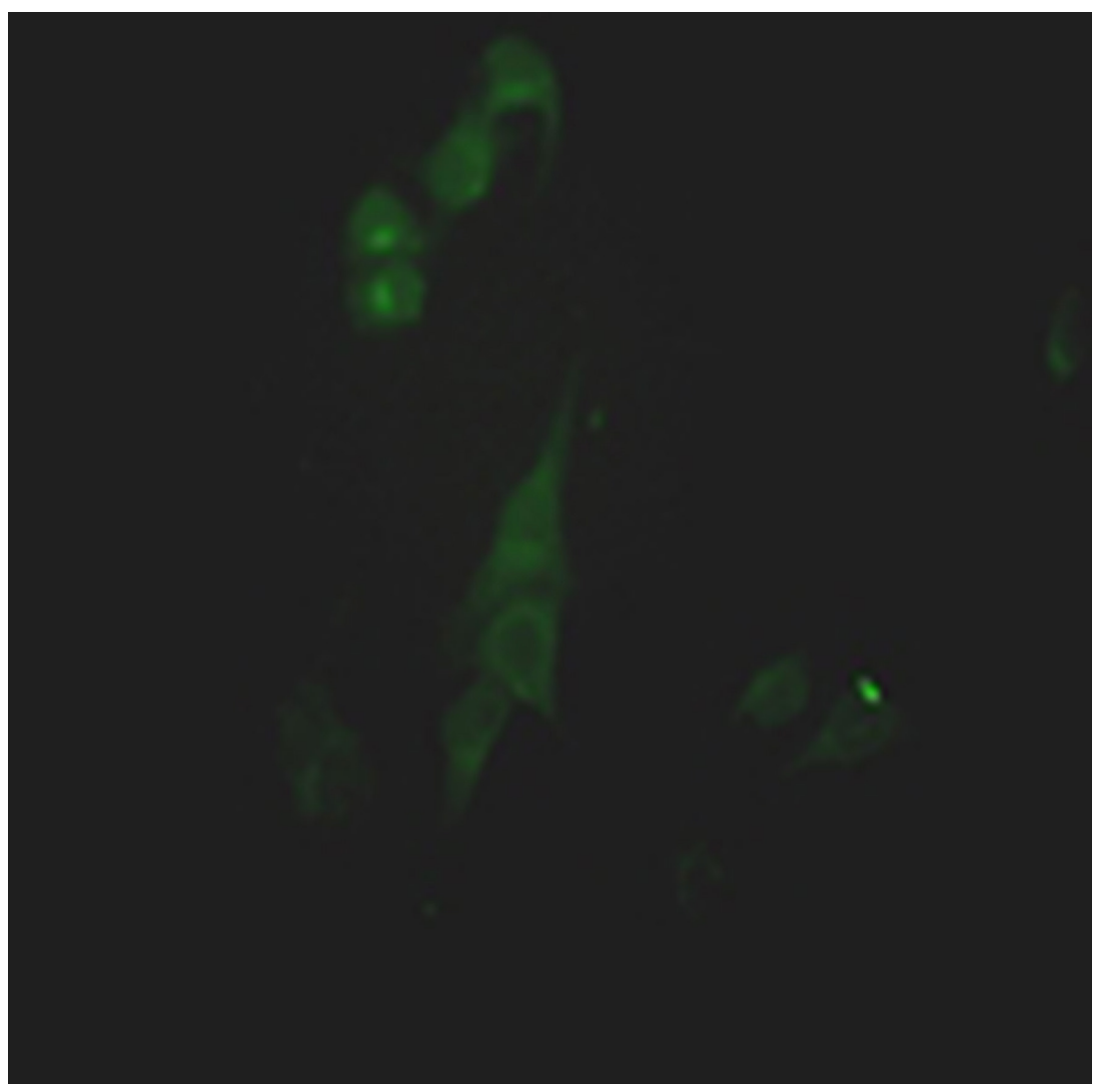

Figure 2. Indirect immunofluorescence staining of melanocytes in serum samples obtained from healthy subjects (40X).

\section{Identification of vitiligo-associated membrane antigen by mass spectrometry}

The results of the mass spectrometry analysis (Table 1 ) identified the vitiligo-associated membrane antigen with relative molecular weights of $86 \times 10^{3}$ and $75 \times 10^{3}$ as lamin $\mathrm{A} / \mathrm{C}$; while the vitiligo-associated membrane antigen with an $\mathrm{Mr}$ of $60 \times 10^{3}$ was identified as Vimentin X1.

\section{Detection of serum anti-TRP-1 and HSP70 by ELISA}

The serum anti-TRP-1 and HSP70 levels were detected by ELISA, the results of which are summarized in Table 2. One-way ANOVA identified significant differences in the levels of expression of the anti-TRP-1 antibody among the three groups $(F=362.802 ; \mathrm{P}=0.000)$. Further multiple comparison revealed significant differences in the level of expression of anti-TRP-1 in the HBsAg-positive compared to the HBsAg-negative vitiligo group and the control group $(P=0.000)$. However, no significant differences were observed between the HBsAg-negative vitiligo group and the control group $(P=1.000)$. Meanwhile, HSP70 expression differed significantly among the 
three groups $(F=252.722 ; P=0.000)$. Multiple comparisons revealed significant differences in HSP70 expression in the HBsAg-positive vitiligo group compared to that in the HBsAg-negative vitiligo group and the control group $(P=0.000)$. However, no significant differences were observed between HBsAg-negative vitiligo group and the control group ( $P=0.512$ ). The anti-TRP-1 and -HSP70 levels in the 10 vitiligo patients with hepatitis B (HBsAg-positive) were positively correlated $(r=0.927 ; P=0.000 ;$ Figure 7$)$.

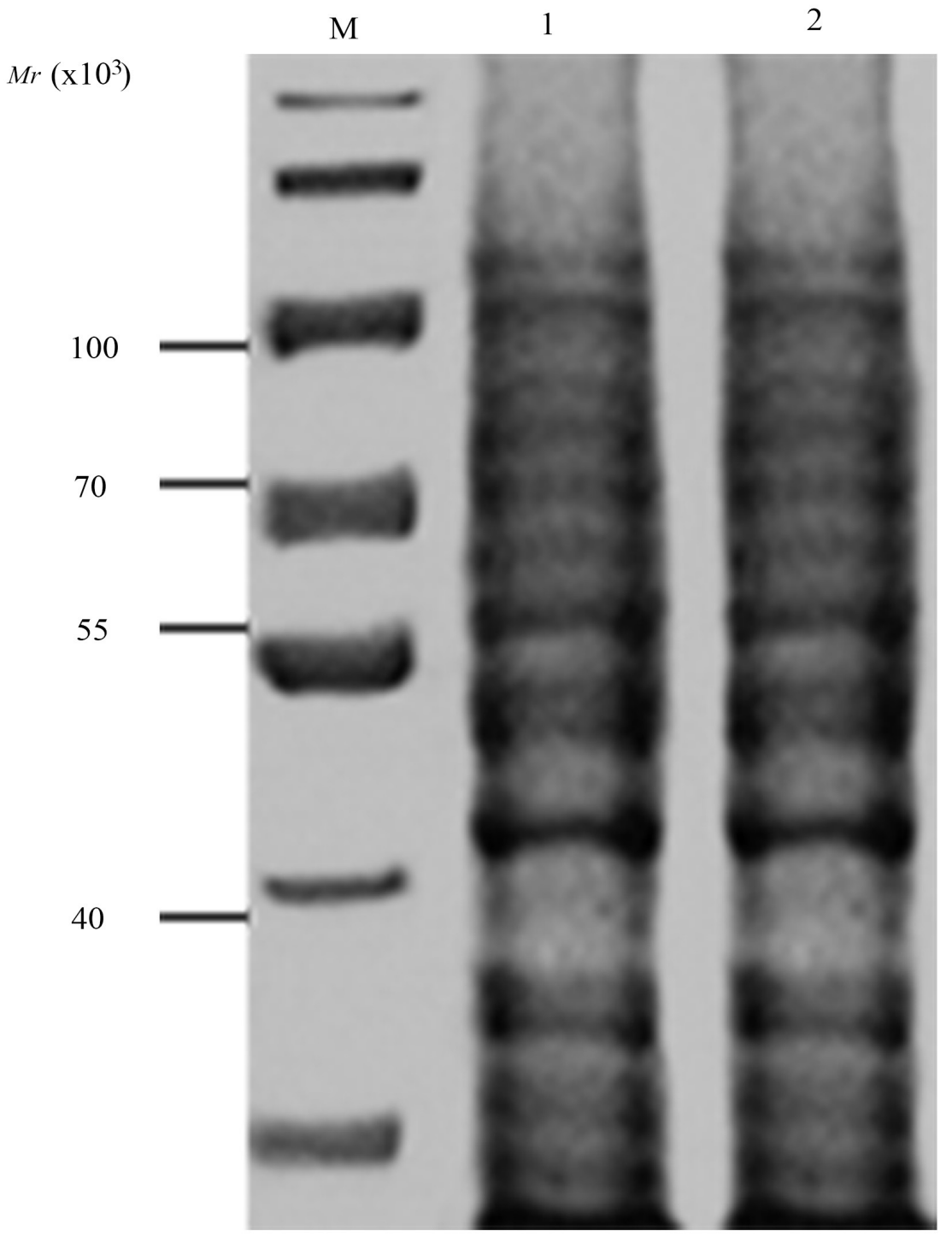

Figure 3. Sodium dodecyl sulfate polyacrylamide gel electrophoresis (SDS-PAGE) of melanocyte cytoplasmic proteins. Lane $M=$ protein marker; lanes 1-2 = cytoplasmic proteins extracted from melanocytes. 


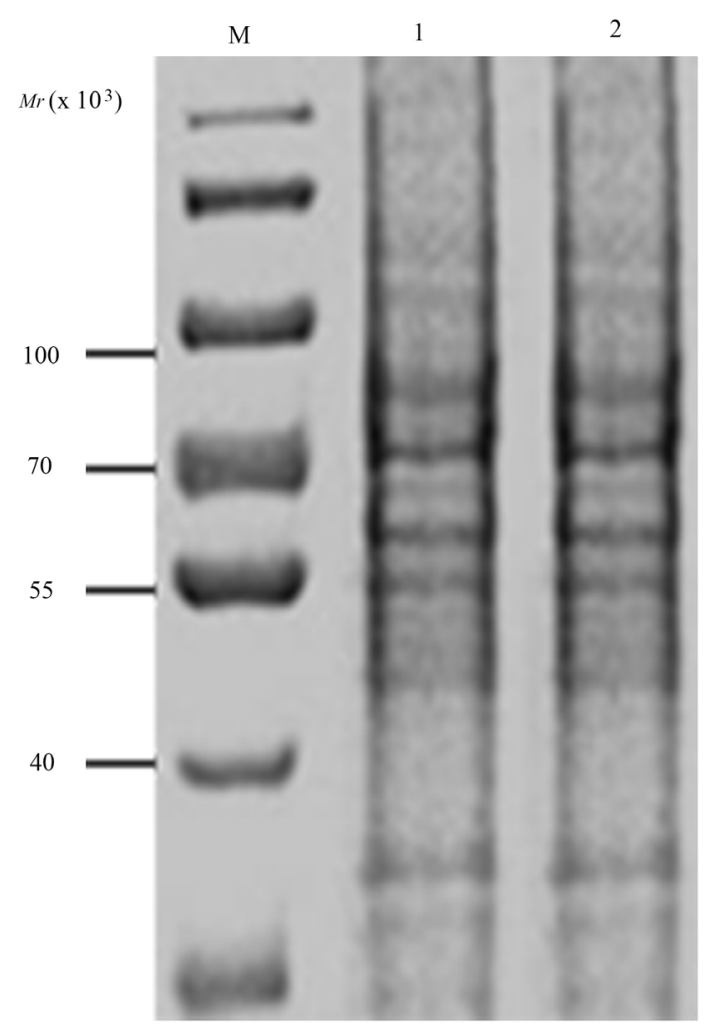

Figure 4. SDS-PAGE analysis of melanocyte membrane proteins. Lane M: protein marker; lanes 1-2: membrane proteins extracted from melanocytes.

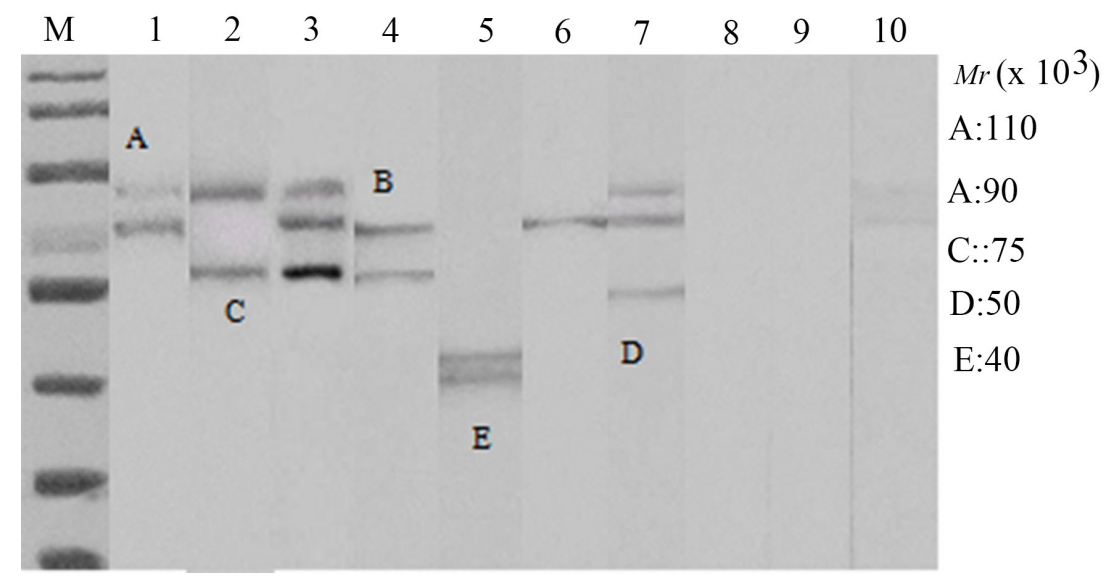

Figure 5. Western blot analysis of membrane antigens. Lane M: protein marker; lanes 1-7: strong positive results from sera obtained from 7 vitiligo patients; lanes 8-10: control sera. 


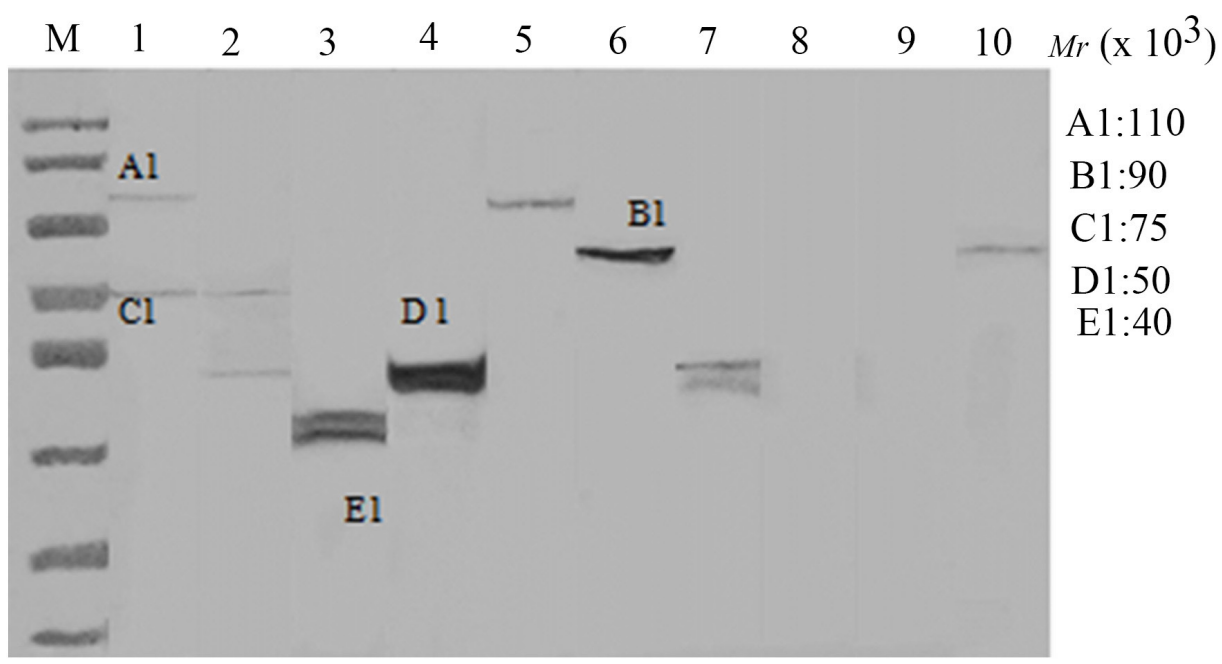

Figure 6. Western blot analysis of cytoplasmic antigens. Lane M: protein marker; lanes 1-7: strong positive results from sera obtained from 7 vitiligo patients; lanes 8-10: control sera.

Table 1. Identification of melanocyte membrane antigens by protein mass spectrometry.

\begin{tabular}{|c|c|c|c|c|c|c|c|c|}
\hline $\begin{array}{l}\text { Target } \\
\text { protein }\end{array}$ & $\begin{array}{l}\text { Serial } \\
\text { number }\end{array}$ & $\begin{array}{c}\text { Theoretical } \\
\text { Mr }\end{array}$ & $\begin{array}{c}\text { Theoretical } \\
\text { pl }\end{array}$ & $\begin{array}{c}\text { Coverage } \\
\text { rate }(\%)\end{array}$ & Score & $\begin{array}{c}\text { Specific } \\
\text { polypeptide }\end{array}$ & $\begin{array}{c}\text { Total } \\
\text { amino acid }\end{array}$ & Total \\
\hline \multicolumn{9}{|l|}{$86 \times 10^{3}$} \\
\hline Lamin A & 27436946 & 74.1 & 7.02 & 51.66 & 280.72 & 2 & 124 & 664 \\
\hline Lamin C & 5031875 & 65.1 & 6.84 & 56.82 & 273.70 & 1 & 118 & 572 \\
\hline \multicolumn{9}{|l|}{$75 \times 10^{3}$} \\
\hline Lamin C & 5031875 & 65.1 & 6.84 & 56.82 & 444.43 & 1 & 189 & 572 \\
\hline Lamin A & 27436946 & 74.1 & 7.02 & 47.59 & 399.09 & 1 & 179 & 664 \\
\hline \multicolumn{9}{|l|}{$60 \times 10^{3}$} \\
\hline Vimentin X1 & 578818565 & 53.6 & 5.12 & 49.14 & 271.73 & 20 & 130 & 466 \\
\hline
\end{tabular}

$\mathrm{Mr}=$ relative molecular weight.

Table 2. Detection of anti-TRP-1 antibody and heat shock protein 70 expression using enzyme-linked immunosorbent assay (ELISA).

\begin{tabular}{|c|c|c|c|}
\hline \multirow[t]{2}{*}{ Group } & \multirow[t]{2}{*}{ Number of samples $(\mathrm{N})$} & \multicolumn{2}{|c|}{ A value (means $\pm S D$ ) } \\
\hline & & anti-TRP-1 antibody & HSP70 \\
\hline HBsAg $(+)$ vitiligo group & 10 & $1.754 \pm 0.481$ & $1.669 \pm 0.487$ \\
\hline HBsAg (-) vitiligo group & 60 & $0.167 \pm 0.075$ & $0.238 \pm 0.112$ \\
\hline Control group & 26 & $0.169 \pm 0.096$ & $0.206 \pm 0.107$ \\
\hline
\end{tabular}

HBsAg, hepatitis B infected; A value, absorbance; TRP-1, anti-tyrosinase-related protein; HSP70, heat shock protein 70; SD, standard deviation. 


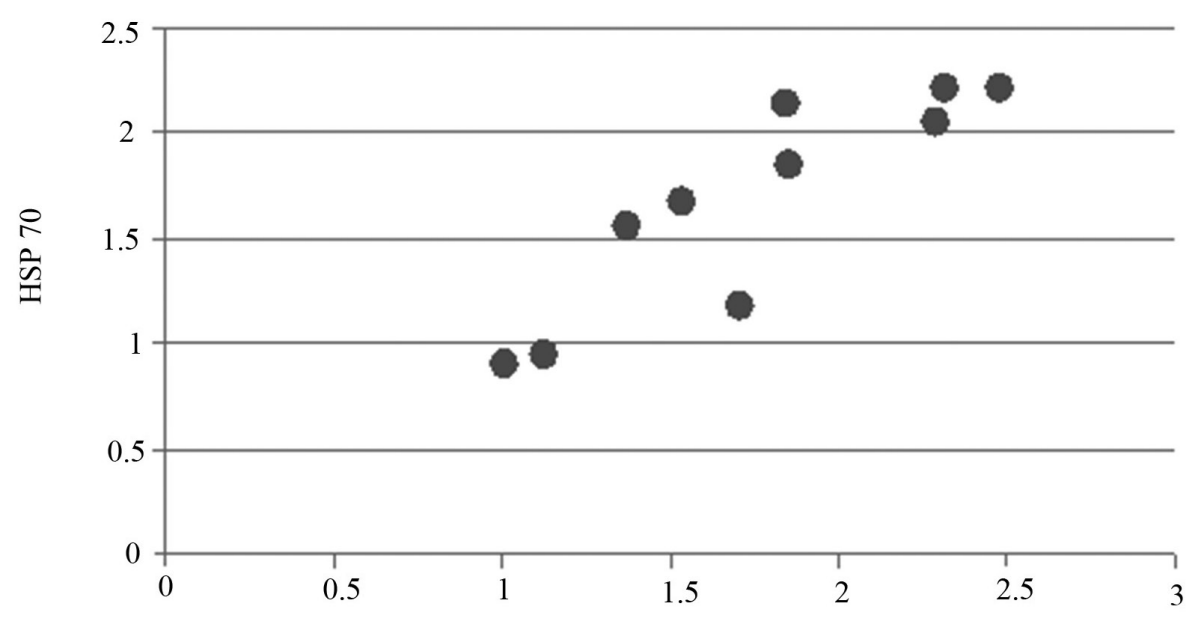

Anti-TRP-lantibody

Figure 7. Correlation analysis of anti-TRP-1 antibodies and heat shock protein 70 .

\section{DISCUSSION}

The existence of anti-melanocyte antibody in the serum of vitiligo patients was first reported by Naughton et al. (1983). In 1992, Cui et al. identified autoantibodies against one or several membrane antigens (Mr: $35 \times 10^{3}, 40 \sim 45 \times 10^{3}, 75 \times 10^{3}, 90 \times 10^{3}$, and $150 \times 10^{3}$ ) on melanocytes. On the other hand, Hann et al. (1996) discovered the molecular weights of the antigens targeted by these antibodies to be approximately $45 \times 10^{3}, 65 \times 10^{3}, 80 \times 10^{3}$, and $110 \times$ $10^{3}$ by immunoblotting. The characteristics of the vitiligo-associated antigens detected in this study were generally consistent with those seen in previous studies. However, the antigens with relative molecular weights $35 \times 10^{3}$ and $150 \times 10^{3}$ were not observed in this study.

$\mathrm{Li}$ et al. (2011) reported the separation of melanocyte membrane antigens by twodimensional electrophoresis; subsequently, the immune staining spots shown by vitiligo patients (serum samples showed strong-positive staining against melanocytes) and healthy controls were compared and analyzed. The membrane antigens with $\mathrm{Mr} 75 \times 10^{3}, 60 \times 10^{3}$, and $45 \times 10^{3}$ were identified as lamin A/C, TRP-1, and melanin-concentrating hormone receptor 1 , respectively. In addition, the membrane antigen with an $\mathrm{Mr}$ of $75 \times 10^{3}$ was specifically identified as lamin $\mathrm{A}$, an important skeleton protein existing in the cell nucleus. Nuclear laminar proteins play an important role in maintaining the morphological structures of cells. These proteins were sub-grouped into lamin A, B, and C. Anti-lamin antibodies, which are anti-nuclear antibodies, have been previously observed in the serum of patients with autoimmune diseases (Wesierska-Gadek et al., 1988; Senecal et al., 1999; Nesher et al., 2001; Coppo et al., 2004), and have been used to screen for diseases such as autoimmune hepatitis (lamin A and $C$ ) and systemic lupus erythematosus (SLE; lamin B). In this study, the identification of the $\mathrm{Mr} 75 \times 10^{3}$ membrane antigen was consistent with the result obtained by Li et al. (2011). However, the $86 \times 10^{3}$ membrane antigen was also identified as lamin $A$; the difference in bands may be attributed to protein modification, causing a slight difference in the Mr. This could also explain the appearance of other comparable bands, such as the membrane antigens with $\mathrm{Mr}$ of $44 \times 10^{3}, 40 \times 10^{3}$, and $50 \times 10^{3}$. 
On the other hand, in this study, the membrane antigen with an $\mathrm{Mr}$ of $60 \times 10^{3}$ was identified as vimentin $\mathrm{X} 1$, a newly discovered vitiligo-associated membrane antigen. Vimentin, a type of intermediate filament, is also a component of the cytoskeleton, which connects the nuclear and bridge corpuscles on the cell surface, and maintains the spatial location of the nucleus and organelles within the cells. A high titer of autoimmune hepatitis can be detected in a variety of autoimmune diseases, such as autoimmune hepatitis, SLE, and systemic scleroderma. Additionally, previous studies (Cheng et al., 2008, Liu et al., 2012) have reported that anti-mutated citrullinated vimentin antibodies are highly sensitive and specific in rheumatoid arthritis.

Autoimmune antibodies are generally believed to damage the melanocytes via the complement lysis effect or antibody-dependent cell-mediated cytotoxicity. A majority of the researchers believe that autoimmune antibodies exert an effect by combining with their corresponding membrane antigens. However, Isenberg et al. (1997) reported that antibodies internalized by non-hematopoietic cells in the human body could also react with nuclear antigens, suggesting the possibility of melanocytes constantly and actively taking up antibodies in order to elicit appropriate immune responses. In this study, the $75 \times 10^{3}$ and $60 \times 10^{3}$ antigens were both located within the cell nucleus, supporting the theory that vitiligo was a result of the damage caused by anti-nuclear antibodies taken up by melanocytes.

$\mathrm{Li}$ et al. (2011) identified at least one concomitant autoimmune disease in approximately half of the vitiligo patients expressing the anti-lamin A antibody $(+)$. Therefore, the anti-lamin A antibody was believed to be one of the major markers for the identification and diagnosis of such patients. The immune responses against self-antigens, developed as a result of the HBV infection, induced the production of various autoantibodies, which in turn led to the development of autoimmune diseases (Wang et al., 2010). Our experiments focusing on vitiligo-associated cytoplasmic antigens revealed a high expression of the anti-TRP-1 antibody in the serum of vitiligo patients with hepatitis B; on the other hand, the level of expression of the anti-TRP-1 antibody in patients without hepatitis B was found to be quite low. Moreover, immunoblotting analysis allowed for the identification of antibodies against melanocyte-associated antigens ( $\mathrm{Mr} 75 \times 10^{3}$ and $60 \mathrm{x}$ $\left.10^{3}\right)$ in the serum of all patients with concomitant hepatitis $B(N=4)$. These results further confirm the relationship between autoimmune diseases and vitiligo pathogenesis.

In 2011, Kim et al. utilized protein mass spectrometry to identify HSP70, a significant signal transduction molecule participating in the antigen presentation process, as a specific vitiligoassociated protein. Serum samples from patients with some (other) autoimmune diseases have shown the presence of autoantibodies targeting HSP70; the overexpression of HSP70 usually results in the initiation of autoimmune response against melanocytes (Denman et al., 2008). HBV infection results in cytotoxic T lymphocyte-mediated liver cell damage, which in turn leads to the upregulation of serum HSP70, which could cause the production of anti-melanocyte antibodies and the initiation of an autoimmune response against self-melanocytes. In this study, we identified a significant positive correlation between the level of HSP70 and anti-TRP-1 antibody expression in the serum of vitiligo patients with hepatitis B; this indicated that HSP70, together with a variety of other factors, may cause the development of vitiligo in patients infected with HBV.

Research on vitiligo-associated melanocyte antigens has greatly progressed over the past few years. Investigation and identification of these autoantigens and their epitopes (that are recognized by autoantibodies) are of great importance to facilitate the elucidation of vitiligo pathogenesis and its treatment. Membrane antigens with a relatively high positive expression rate ( $\mathrm{Mr} 60 \times 10^{3}, 75 \times 10^{3}$, and $86 \times 10^{3}$ ) have been identified in this study. However, other antigens, especially cytoplasmic antigens, must be further investigated and identified in the future. 


\section{Conflicts of interest}

The authors declare no conflict of interest.

\section{ACKNOWLEDGMENTS}

Research supported by grants provided by the Application Research of Clinical Characteristics of the Capital (\#Z111107058811086).

\section{REFERENCES}

Cheng P, Zhong LM, Shen J, Li Zl, et al. (2008). Detection of the antibodies of anti-mutated citrullinated vimentin in diagnosis of rheumatoid arthritis. Chin. J. Immunol. 24: 1028-1032.

Coppo P, Clauvel JP, Bengoufa D, Fuentes V, et al. (2004). Autoimmune cytopenias associated with autoantibodies to nuclear envelope polypeptides. Am. J. Hematol. 77: 241-249.

Cui J, Harning R, Henn M and Bystryn JC (1992). Identification of pigment cell antigens defined by vitiligo antibodies. J. Invest. Dermatol. 98: 162-165.

Cui J, Arita Y and Bystryn JC (1993). Cytolytic antibodies to melanocytes in vitiligo. J. Invest. Dermatol. 100: $812-815$.

Denman CJ, McCracken J, Hariharan V, Klarquist J, et al. (2008). HSP70i accelerates depigmentation in a mouse model of autoimmune vitiligo. J. Invest. Dermatol. 128: 2041-2048.

Hann SK, Shin HK, Park SH, Reynolds SR, et al. (1996). Detection of antibodies to melanocytes in vitiligo by western immunoblotting. Yonsei Med. J. 37: 365-370.

Isenberg D, Rahman MA, Ravirajan CT and Kalsi JK (1997). Anti-DNA antibodies: from gene usage to crystal structures. Immunol. Today 18: 149-153.

Kemp EH, Gavalas NG, Gawkrodger DJ and Weetman AP (2007). Autoantibody responses to melanocytes in the depigmenting skin disease vitiligo. Autoimmun. Rev. 6: 138-142.

Kim JY, Do JE, Ahn KJ, Noh S, et al. (2011). Detection of melanocyte autoantigens reacting with autoantibodies in vitiligo patients by proteomics. J. Dermatol. Sci. 62: 202-204.

Li Q, Gao TT, Li CY, Lu T, et al. (2003). Detection of melanocyte membrane antigen associated with vitiligo. Chin. J. Dermatol. 36: 76-78.

Li Q, Lv YJ, Li CY, Yi X, et al. (2011). Vitiligo autoantigen VIT75 is identified as lamin A in vitiligo by serological proteome analysis based on mass spectrometry. J. Invest. Dermatol. 131: 727-734.

Li ZQ, Gao TW, Ma CL, Chen JM, et al. (2000). Detection of anti-melanocyte IgG and IgM antibody in the serum of vitiligo patients and complement mediated serum cytotoxicity reaction. J. Clin. Dermatol. 3: 136-138.

Li ZQ, Liu RQ, Gao TW, Zhao XD, et al. (2001). Detection of anti-melanocyte membrane antigen autoantibody in the serum of vitiligo patients. Chin. J. Dermatol. 34: 268-270.

Liu LJ, Jin HT and Liu H (2012). Clinic evaluation of anti-mutated citrullinated vimentin in early rheumatoid arthritis. Xinjiang Med. J. 42: 5-8.

Lu T, Gao TW, Wang AH, Li Q, et al. (2004). The prevalence of vitiligo in Shaanxi Province of China. Chin. J. Dermatol. 37: 406-407.

Mulekar SV (2003). Melanocyte-keratinocyte cell transplantation for stable vitiligo. J. Invest. Dermatol. 42: $132-136$.

Naughton GK, Eisinger M and Bystryn JC (1983). Detection of antibodies to melanocytes in vitiligo by specific immunoprecipitation. J. Invest. Dermatol. 81: 540-542.

Nesher G, Margalit R and Ashkenazi YJ (2001). Anti-nuclear envelope antibodies: clinical associations. Semin. Arthritis Rheum. 30: 313-320.

Senecal JL, Rauch J, Grodzicky T, Raynauld JP, et al. (1999). Strong association of autoantibodies to human nuclear lamin B1 with lupus anticoagulant antibodies in systemic lupus erythematosus. Arthritis Rheum. 42: 1347-1353.

Wang LL and Zhu XJ (2011). The study on culture condition and biological identification for human epidermal melanocytes in vitro. J. Diagn. Ther. Dermato-Venereol. 18: 87-90.

Wang R, Geng XR, Hong H, Tan TC, et al. (2010). Features of autoantibodies in patients with hepatitis B. Practical Clin. J. Integrated Tradit. Chin. West Med. 7: 85-87.

Wesierska-Gadek J, Penner E, Hitchman E and Sauermann G (1988). Antibodies to nuclear lamins in autoimmune liver disease. Clin. Immunol. Immunopathol. 49: 107-115. 
Zhang YJ, Liu L, Li Q, Li C, et al. (2012). Clinical profiles of vitiligo in China: an analysis of 10821 patients with association diseases. Chin. J. Aesthetic Med. 21: 1589-1592.

Zhou MH, Lu Y, Li X, Wu D, et al. (2013). Transplantation of cultured autologous melanocytes for the treatment of vitiligo. China Med. Cosmetol. 3(3): 78-79.

Zhu MC, Ma HY, Zhan Z, Wang H, et al. (2010). Immunofluorescence test of serum antibody against melanocytes in vitiligo patients. Med. J. Nat. Defending Forces in Southwest China 20: 29-31. 